\title{
Secondary Small Interfering RNA-Based Silencing Tools in Plants: An Update
}

\author{
Alberto Carbonell* \\ Instituto de Biología Molecular y Celular de Plantas, Consejo Superior de Investigaciones Científicas-Universitat Politècnica \\ de València, Valencia, Spain
}

Keywords: RNA silencing, secondary siRNA, tasiRNA, atasiRNA, syn-tasiRNA, MIGS

\section{PLANT SECONDARY SMALL INTERFERING RNAS}

In plants, RNA silencing regulates key biological processes such as development, response to stress, genome integrity, and antiviral resistance. RNA silencing functions through diverse classes of small RNAs (sRNAs) that associate with ARGONAUTE (AGO) proteins to repress highly sequence-complementary target transcripts (Baulcombe, 2004).

Small interfering RNAs (siRNAs) are a class of sRNAs arising from double-stranded RNA

OPEN ACCESS

Edited by:

Andrea Chini,

Centro Nacional de Biotecnología

(CNB), Spain

Reviewed by:

German Martinez,

Swedish University of Agricultural

Sciences, Sweden

Blake Meyers,

Donald Danforth Plant Science Center,

United States

*Correspondence:

Alberto Carbonell

acarbonel/@ibmcp.upv.es

Specialty section:

This article was submitted to

Plant Physiology,

a section of the journal

Frontiers in Plant Science

Received: 28 March 2019

Accepted: 07 May 2019

Published: 28 May 2019

Citation:

Carbonell A (2019) Secondary Small Interfering RNA-Based Silencing Tools

in Plants: An Update.

Front. Plant Sci. 10:687.

doi: $10.3389 / f p / s .2019 .00687$
(dsRNA) precursors. Secondary siRNAs are those siRNAs whose dsRNA precursor synthesis is triggered by an upstream sRNA-guided transcript cleavage event followed by RNA-dependent RNA polymerase (RDR) activity (for a recent review see de Felippes, 2019). Many secondary siRNAs are produced in 21-nucleotide (nt) register with the sRNA-guided cleavage site by successive Dicer-Like (DCL) processing and are therefore called phased secondary siRNAs (phasiRNAs). In contrast, only a subset of secondary siRNAs act in trans to repress one or more targets distinct from their locus of origin. These siRNAs are called trans-acting siRNAs (tasiRNAs), most of which are also phased (Axtell, 2013).

\section{CLASSES OF SECONDARY SIRNA-BASED SILENCING TOOLS}

While secondary siRNAs may be ultimately generated in classic RNA interference (RNAi) approaches such as virus induced gene silencing (VIGS) or hairpin-based silencing after the initial targeting of transgene-derived (primary) siRNAs (Ossowski et al., 2008), only two classes of silencing tools operate primarily through the action of secondary siRNAs. These are (i) artificial or synthetic tasiRNAs (atasiRNAs and syn-tasiRNAs, respectively, both terms are accepted), and (ii) miRNA-induced gene silencing (MIGS). Both classes of secondary siRNA-based tools have been extensively used in plants to induce selective gene silencing in basic gene function studies or to improve agronomic traits.

atasiRNA/syn-tasiRNAs are expressed from transgenes including engineered TAS precursors in which a region corresponding to various endogenous tasiRNAs is substituted by a fragment containing multiple atasiRNA/syn-tasiRNA sequences (Figure 1A). In Arabidopsis thaliana (Arabidopsis), modified TAS transcripts are cleaved by a specific microRNA (miRNA)/AGO complex (e.g., miR173/AGO1 and miR390/AGO7 cleave TAS1- and TAS3-based precursors, respectively), and one of the cleavage products is converted by RDR6 to dsRNA, which is processed by DCL4 into phased tasiRNA duplexes in 21-nt register with the miRNA cleavage site. atasiRNA/syn-tasiRNA guide strands, typically designed to contain an AGO1-preferred 5' $\mathrm{U}$, are incorporated into AGO1 to direct silencing of one or multiple transcripts at one or multiple sites (Figure 1A). Importantly, the multiplexing of several atasiRNAs/syn-tasiRNAs in 


\section{A}

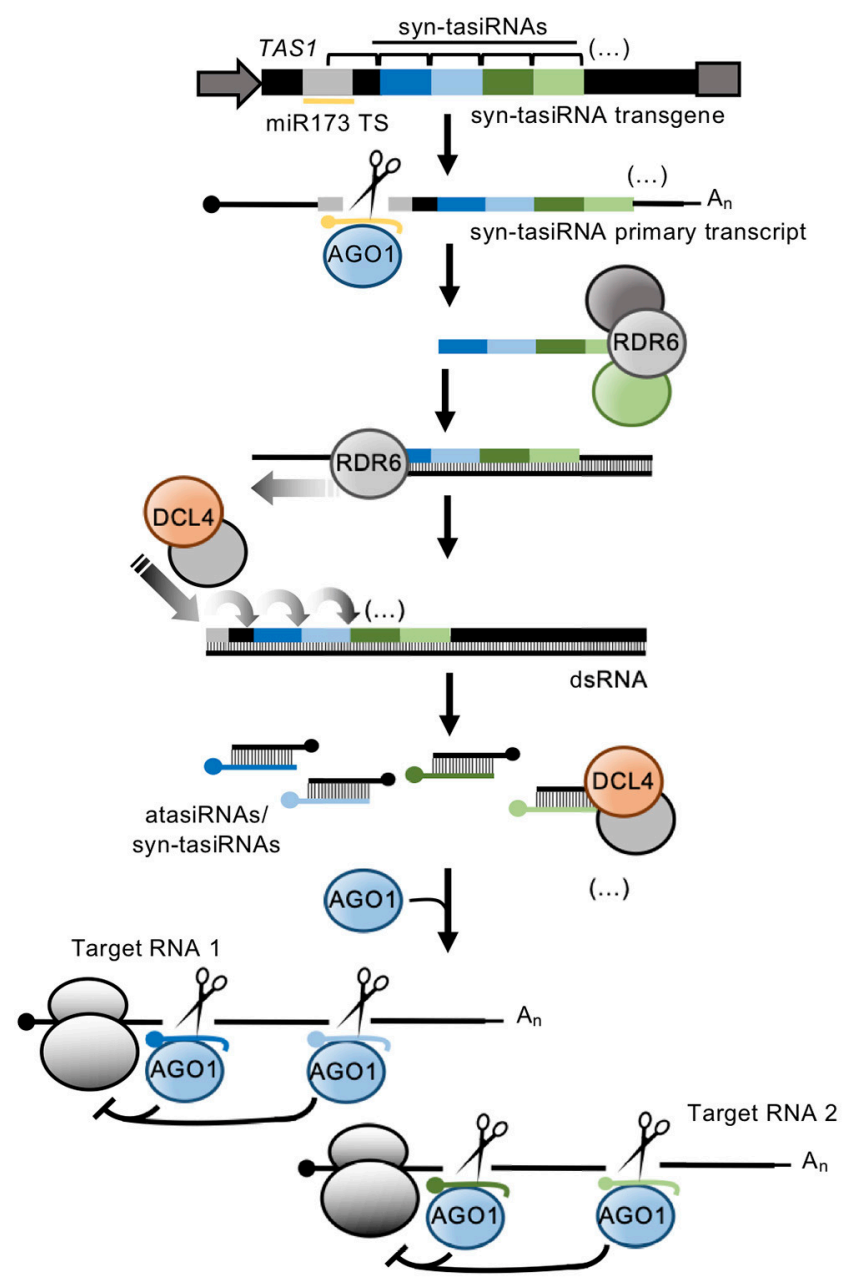

B

MIGS
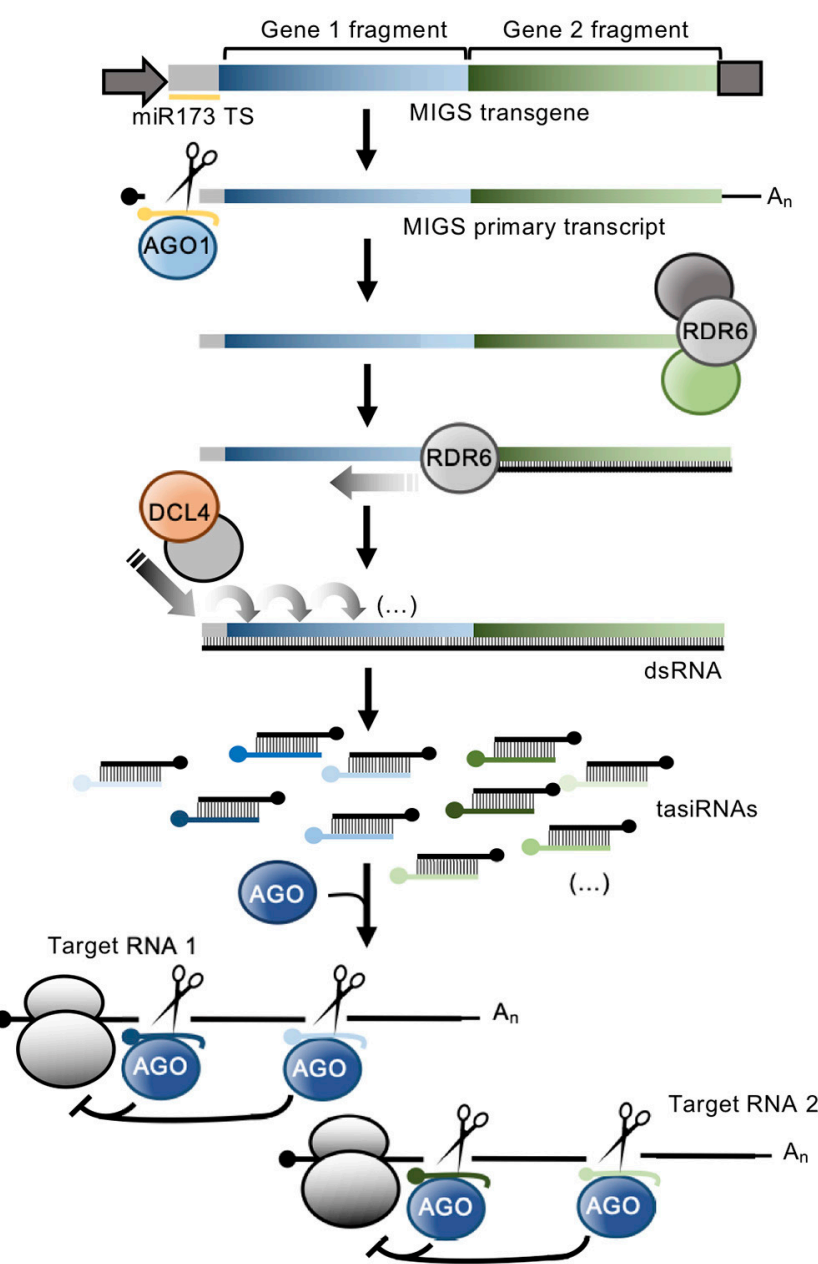

FIGURE 1 | Secondary siRNA-based silencing tools in plants. (A). The atasiRNA/syn-tasiRNA pathway. TAS1 precursor sequence is in black, with trigger miR173 target site (TS) in light gray. AtasiRNA/syn-tasiRNA sequences targeting RNA 1 are shown in dark and light blue; atasiRNA/syn-tasiRNA sequences targeting RNA 2 are shown in dark and light green; miR173 sequence is shown in yellow. Promoter and terminator sequences are shown with a dark gray arrow and box, respectively. Participating proteins are represented with colored ovals. (B) The MIGS pathway. Sequences corresponding to gene fragments 1 and 2 are in diverse blue and green tonalities, respectively. Other details are as in (A)

a single construct allows for the efficient and simultaneous multitargeting of various sequence-related or unrelated genes. Moreover, as for artificial miRNAs (amiRNAs), atasiRNAs/syntasiRNAs can be computationally designed with user-friendly web tools such as P-SAMS (http://p-sams.carringtonlab.org/) (Fahlgren et al., 2016) to be highly specific and prevent the socalled off-target effects characteristic of other RNAi approaches. For instance, P-SAMS designs artificial sRNAs that contain (i) an AGO1-preferred 5' U, (ii) a C in position 19 to generate a star strand with an AGO1 non-preferred 5' G thus avoiding competition for AGO1 loading, and (iii) an intentional mismatch with the target transcript at position 21 to limit possible transitivity effects (Carbonell, 2017). Initially, atasiRNAs/syntasiRNAs were used to efficiently repress one or multiple endogenous genes in gene function studies in Arabidopsis (de La Luz Gutierrez-Nava et al., 2008; Montgomery et al., 2008a,b; Carbonell et al., 2014) (Table 1). More recently, atasiRNAs/syntasiRNAs have emerged as an effective approach to induce resistance against viruses and viroids in several plant species (Chen et al., 2016; Carbonell and Daròs, 2017; Carbonell et al., 2019) (Table 1), and, more broadly, as a promising tool for plant biology study and crop improvement (for a review see Zhang, 2014).

MIGS was named (de Felippes et al., 2012) a few years later than was first reported (Montgomery et al., 2008b; Felippes and Weigel, 2009). Initial MIGS transgenes included one or more fragments from one or more target genes fused downstream of miR173 target site (Figure 1B) (de Felippes 
TABLE 1 | Uses of secondary siRNA-based tools in plants.

\begin{tabular}{|c|c|c|c|c|c|}
\hline Secondary siRNA tool & Precursor & $\begin{array}{l}\text { miRNA } \\
\text { trigger }\end{array}$ & Plant species & Target(s) ${ }^{a}$ & References \\
\hline \multirow[t]{7}{*}{ atasiRNA/syn-tasiRNA } & TAS1a & miR173 & Arabidopsis thaliana & SUL & $\begin{array}{l}\text { Felippes and Weigel, } 2009 \\
\text { Baykal et al., } 2016\end{array}$ \\
\hline & TAS1C & & & $\begin{array}{l}C P C, E T C 2, \\
F T, T R Y\end{array}$ & Carbonell et al., 2014 \\
\hline & & & & FAD2 & de La Luz Gutierrez-Nava et al., 2008 \\
\hline & & & Nicotiana benthamiana & PSTVd & Carbonell and Daròs, 2017 \\
\hline & & & & TSWV & Carbonell et al., 2019 \\
\hline & TAS3 & miR390 & Arabidopsis thaliana & PDS & Montgomery et al., 2008a \\
\hline & & & & CMV, TuMV & Chen et al., 2016 \\
\hline \multirow[t]{10}{*}{ MIGS } & - & miR173 & Arabidopsis thaliana & $\begin{array}{l}\text { AG, ELF3, } \\
F T, G F P, L F Y\end{array}$ & de Felippes et al., 2012 \\
\hline & & & & PDS & Sarrion-Perdigones et al., 2013 \\
\hline & & & & PGDH1 & Benstein et al., 2013 \\
\hline & & & & PSAT1 & Wulfert and Krueger, 2018 \\
\hline & & & Capsella rubella & RPP5 & Sicard et al., 2015 \\
\hline & & & Medicago truncatula & CEP1 & Imin et al., 2013 \\
\hline & & & Oryza sativa & $\begin{array}{l}\text { GBSS, LAZY1, } \\
\text { PDS, ROC5 }\end{array}$ & Zheng et al., 2018 \\
\hline & & & Petunia hybrida & $C H S, P D S$ & Han et al., 2015 \\
\hline & & miR390 & Arabidopsis thaliana & $\mathrm{CH} 42$ & Felippes and Weigel, 2009 \\
\hline & & & $\begin{array}{l}\text { Nicotiana tabacum } \\
\text { Solanum lycopersicum }\end{array}$ & $\begin{array}{l}\text { TOLCNDV, } \\
\text { TOLCGV }\end{array}$ & Singh et al., 2015 \\
\hline
\end{tabular}

${ }^{a}$ AG, AGAMOUS; CEP1, C-TERMINALLY ENCODED PEPTIDE 1; CH42, CHLORINA 42; CHS, CHALCONE SYNTHASE; CMV, CuCUMber mOsaiC viruS; CPC, CAPRICE; ELF3, EARLY FLOWERING 3; ETC2, ENHANCER OF TRIPTYCHON AND CAPRICE 2; FAD2, $\triangle(12)$-FATTY-ACID DESATURASE; FT, FLOWERING LOCUS T; GBSS, GRANULE BOUND STARCH SYNTHASE 1; GFP, GREEN FLUORESCENT PROTEIN; LAZY1, shoot gravitropism gene; LFY, LEAFY; miP1a, microProtein 1a; miP1b, microProtein 1b; NFR1 $\alpha$, NODULATION FACTOR KINASE 1 $\alpha$; P450 CYP51G1, putative cytochrome P450 CYP51G1; PDS, PHYTOENE DESATURASE; PGDH1, PHOSPHOGLYCERATE DEHYDROGENASE 1; PPV, Plum pOX virus; PSAT1, PHOSPHOSERINE AMINOTRANSFERASE 1; PSTVd, Potato spindle tuber viroid; ROC5, RICE OUTERMOST CELL-SPECIFIC 5; RPP5, RECOGNITION OF PERONOSPORA PARASITICA 5; SUL, SULFUR; ToLCGV, Tomato leaf curl Gujarat virus; ToLCNDV, Tomato leaf curl New Delhi virus; TRY, TRIPTYCHON; TSWV, Tomato spotted wilt virus; TuMV, Turnip mosaic virus.

et al., 2012). miR173, as other 22 nt miRNAs, possesses the ability of triggering the production of phasiRNAs from target transcripts (Chen et al., 2010; Cuperus et al., 2010). In MIGS, miR173/AGO1-guided cleavage of the MIGS primary transcript triggers RDR6-dependent synthesis of dsRNA which is subsequently processed by DCL4 to release phased tasiRNAs that lead to the efficient silencing of target genes (Figure 1B). Interestingly, MIGS can also be triggered by other 22-nt miRNAs such as miR1514a.2 (Jacobs et al., 2016), or by miR390 (Felippes and Weigel, 2009; Singh et al., 2015), a 21-nt miRNA with unique properties for triggering tasiRNA formation from TAS3 transcripts (Montgomery et al., 2008a) (Table 1). Because miR173 is present only in Arabidopsis and closely-related species, miR173 co-expression with MIGS transgenes is necessary to trigger tasiRNA biogenesis in non-Arabidopsis species as reported in Medicago truncatula (Imin et al., 2013), Petunia (Han et al., 2015), soybean (Jacobs et al., 2016), and rice (Zheng et al., 2018). Despite having been widely used in gene function studies and also to confer antiviral resistance (Table 1), the MIGS approach presents a significant risk of off-target effects due to (i) the large number of tasiRNAs generated from the MIGS construct (similarly to those from classic RNAi constructs), (ii) the generation of out-of-phase siRNAs from MIGS constructs that can accumulate to high levels as observed in Petunia (Han et al., 2015), and (iii) the possibility that MIGS-derived tasiRNAs induce transitivity as reported (Han et al., 2015). Finally, loading of MIGS-derived tasiRNA into particular AGOs cannot be controlled, and for instance only a subset of these tasiRNAs, typically those with a 5' U, will be loaded into AGO1 while others may be loaded into different AGOs or degraded. 


\section{APPROPRIATE TERMINOLOGY TO REFER TO SECONDARY SIRNA-BASED SILENCING TOOLS}

After having reviewed the literature, it seems necessary to make some brief remarks to improve the proper and consistent use of the terminology related to these secondary siRNA-based tools. For example, the production of tasiRNAs from a transgene including a gene fragment fused downstream to a miRNA trigger target site should always be referred to as MIGS, and not to as atasiRNA/syn-tasiRNA as observed in several works (Singh et al., 2015; Zhao et al., 2015; Guo et al., 2016), even if the MIGS cassette is inserted into a TAS precursor (Zhao et al., 2015; Guo et al., 2016). Also, secondary siRNAs generated from MIGS transgenes should be referred as phasiRNAs or, even better, as tasiRNAs as they are expected to act in trans to target the desired target gene(s), but not as atasiRNAs or syn-tasiRNAs as reported (Singh et al., 2015; Zhao et al., 2015). I suggest to use the term "artificial" or "synthetic" when referring to those transgenederived tasiRNAs that are incorporated into precursors (TAS or others) as individual 21-nt guide sequences that may have been designed computationally to be highly specific in silencing the corresponding target transcript(s), to be preferentially and selectively loaded by AGO1 or to avoid transitivity effects.

\section{CONCLUSIONS AND FUTURE PERSPECTIVES}

Still in the genome editing era dominated by CRISPR/CAS9based technologies, we anticipate that secondary sRNA-based silencing tools will continue to be broadly used because of their unique features in allowing (i) highly specific silencing (e.g., atasiRNAs/syn-tasiRNAs), (ii) the study of genes whose complete knock-out induces lethality, (iii) multitargeting, as well as the targeting of duplicated genes, antisense transcripts and individual isoforms, and (iv) the spatio-temporal control of silencing when transgene expression is regulated with tissue specific or inducible

\section{REFERENCES}

Axtell, M. J. (2013). Classification and comparison of small RNAs from plants. Annu. Rev. Plant Biol. 64, 137-159. doi: 10.1146/annurev-arplant-050312-120043

Baulcombe, D. (2004). RNA silencing in plants. Nature 431, 356-363. doi: $10.1038 /$ nature 02874

Baykal, U., Liu, H., Chen, X., Nguyen, H. T., and Zhang, Z. J. (2016). Novel constructs for efficient cloning of sRNA-encoding DNA and uniform silencing of plant genes employing artificial trans-acting small interfering RNA. Plant Cell Rep. 35, 2137-2150. doi: 10.1007/s00299-0162024-9

Benstein, R. M., Ludewig, K., Wulfert, S., Wittek, S., Gigolashvili, T., Frerigmann, H., et al. (2013). Arabidopsis phosphoglycerate dehydrogenase 1 of the phosphoserine pathway is essential for development and required for ammonium assimilation and tryptophan biosynthesis. Plant Cell 25, 5011-5029. doi: $10.1105 /$ tpc.113.118992

Carbonell, A. (2017). "Artificial small RNA-based strategies for effective and specific gene silencing in plants," in Plant Gene Silencing: promoters. Moreover, as gene knock-down tools, it might be possible to develop secondary siRNA-based strategies for the fine-tuning regulation of secondary siRNA activity to induce the desired degree of target gene silencing.

The atasiRNA/syn-tasiRNA approach seems especially attractive due to its multiplexing capability and high specificity, as well as for the availability of high-throughput cloning strategies and automated design tools for the simple generation of atasiRNA/syn-tasiRNA constructs (Carbonell et al., 2014; Carbonell, 2019). In particular, antiviral atasiRNAs/syn-tasiRNAs designed to target multiple sites in viral RNAs should induce a more effective and durable resistance compared to single target site targeting approaches such as amiRNAs, as the possibility that the virus mutates all target sites to break the resistance seems highly improbable. Still, a deeper knowledge of the basic mechanisms governing secondary siRNA biogenesis, mode of action, and targeting efficacy is needed to further refine these secondary siRNA-based silencing tools in view of accelerating studies of gene function and crop improvement.

\section{AUTHOR CONTRIBUTIONS}

The author confirms being the sole contributor of this work and has approved it for publication.

\section{FUNDING}

This work was supported by grants RYC-2017-21648 and RTI2018-095118-A-100 from the Ministerio de Ciencia, Innovación y Universidades (MCIU, Spain), Agencia Estatal de Investigación (AEI, Spain), and Fondo Europeo de Desarrollo Regional (FEDER, European Union) to AC.

\section{ACKNOWLEDGMENTS}

AC acknowledge support of the publication fee by the CSIC Open Access Publication Support Initiative through its Unit of Information Resources for Research (URICI).
Mechanisms and Applications, ed T. Dalmay (CABI Publishing), 110-127. doi: 10.1079/9781780647678.0110

Carbonell, A. (2019). Design and high-throughput generation of artificial small RNA constructs for plants. Methods Mol. Biol. 1932, 247-260. doi: 10.1007/978-1-4939-9042-9_19

Carbonell, A., and Daròs, J. A. (2017). Artificial microRNAs and synthetic transacting small interfering RNAs interfere with viroid infection. Mol. Plant Pathol. 18, 746-753. doi: 10.1111/mpp.12529

Carbonell, A., López, C., and Daròs, J. A. (2019). Fast-forward identification of highly effective artificial small RNAs against different tomato spotted wilt virus isolates. Mol. Plant Microbe Interact. 32, 142-156. doi: 10.1094/MPMI-05-18-0117-TA

Carbonell, A., Takeda, A., Fahlgren, N., Johnson, S. C., Cuperus, J. T., and Carrington, J. C. (2014). New generation of artificial MicroRNA and synthetic trans-acting small interfering RNA vectors for efficient gene silencing in Arabidopsis. Plant Physiol. 165, 15-29. doi: 10.1104/pp.113.234989

Chen, H. M., Chen, L. T., Patel, K., Li, Y. H., Baulcombe, D. C., and Wu, S. H. (2010). 22-Nucleotide RNAs trigger secondary siRNA biogenesis in plants. Proc. Natl. Acad. Sci. U.S.A. 107, 15269-15274. doi: 10.1073/pnas.1001738107 
Chen, L., Cheng, X., Cai, J., Zhan, L., Wu, X., Liu, Q., et al. (2016). Multiple virus resistance using artificial trans-acting siRNAs. J. Virol. Methods 228, 16-20. doi: 10.1016/j.jviromet.2015.11.004

Cuperus, J. T., Carbonell, A., Fahlgren, N., Garcia-Ruiz, H., Burke, R. T., Takeda, A., et al. (2010). Unique functionality of 22-nt miRNAs in triggering RDR6dependent siRNA biogenesis from target transcripts in Arabidopsis. Nat. Struct. Mol. Biol. 17, 997-1003. doi: 10.1038/nsmb.1866

de Felippes, F. F. (2019). Gene regulation mediated by microRNA-triggered secondary small RNAs in plants. Plants 8:112. doi: 10.3390/plants8050112

de Felippes, F. F., Wang, J. W., and Weigel, D. (2012). MIGS: miRNA-induced gene silencing. Plant J. 70, 541-547. doi: 10.1111/j.1365-313X.2011.04896.x

de La Luz Gutiérrez-Nava, M., Aukerman, M. J., Sakai, H., Tingey, S, V., and Williams, R. W. (2008). Artificial trans-acting siRNAs confer consistent and effective gene silencing. Plant Physiol. 147, 543-551. doi: $10.1104 /$ pp.108.118307

Fahlgren, N., Hill, S. T., Carrington, J. C., and Carbonell, A. (2016). P-SAMS: a web site for plant artificial microRNA and synthetic trans-acting small interfering RNA design. Bioinformatics 32, 157-158. doi: 10.1093/bioinformatics/btv534

Felippes, F. F., and Weigel, D. (2009). Triggering the formation of tasiRNAs in Arabidopsis thaliana: the role of microRNA miR173. EMBO Rep. 10, 264-270. doi: 10.1038/embor.2008.247

Graeff, M., Straub, D., Eguen, T., Dolde, U., Rodrigues, V., Brandt, R., et al. (2016). MicroProtein-mediated recruitment of CONSTANS into a TOPLESS trimeric complex represses flowering in Arabidopsis. PLoS Genet. 12:e1005959. doi: 10.1371/journal.pgen.1005959

Guo, Q., Liu, Q., Smith, N. A., Liang, G., and Wang, M. B. (2016). RNA silencing in plants: mechanisms, technologies and applications in horticultural crops. Curr. Genomics 17, 476-489. doi: 10.2174/1389202917666160520103117

Han, Y., Zhang, B., Qin, X., Li, M., and Guo, Y. (2015). Investigation of a miRNA-induced gene silencing technique in petunia reveals alterations in miR173 precursor processing and the accumulation of secondary siRNAs from endogenous genes. PLOS ONE 10:e0144909. doi: 10.1371/journal.pone. 0144909

Imin, N., Mohd-Radzman, N. A., Ogilvie, H. A., and Djordjevic, M. A. (2013). The peptide-encoding CEP1 gene modulates lateral root and nodule numbers in Medicago truncatula. J. Exp. Bot. 64, 5395-5409. doi: 10.1093/jxb/ert369

Jacobs, T. B., Lawler, N. J., Lafayette, P. R., Vodkin, L. O., and Parrott, W. A. (2016). Simple gene silencing using the trans-acting siRNA pathway. Plant Biotechnol. J. 14, 117-127. doi: 10.1111/pbi.12362

Martínez, G., Panda, K., Köhler, C., and Slotkin, R. K. (2016). Silencing in sperm cells is directed by RNA movement from the surrounding nurse cell. Nat Plants 2:16030. doi: 10.1038/nplants.2016.30

Montgomery, T. A., Howell, M. D., Cuperus, J. T., Li, D., Hansen, J. E., Alexander, A. L., et al. (2008a). Specificity of ARGONAUTE7-miR390 interaction anddual functionality in TAS3 trans-acting siRNA formation. Cell 133, 128-141. doi: 10.1016/j.cell.2008.02.033

Montgomery, T. A., Yoo, S. J., Fahlgren, N., Gilbert, S. D., Howell, M. D., Sullivan, C. M., et al. (2008b). AGO1-miR173 complex initiates phased siRNA formation in plants. Proc. Natl. Acad. Sci. U.S.A. 105, 20055-20062. doi: 10.1073/pnas.0810241105

Ossowski, S., Schwab, R., and Weigel, D. (2008). Gene silencing in plants using artificial microRNAs and other small RNAs. Plant J. 53, 674-690. doi: 10.1111/j.1365-313X.2007.03328.x

Sarrion-Perdigones, A., Vazquez-Vilar, M., Palací, J., Castelijns, B., Forment, J., Ziarsolo, P., et al. (2013). GoldenBraid 2.0: a comprehensive DNA assembly framework for plant synthetic biology. Plant Physiol. 162, 1618-1631. doi: 10.1104/pp.113.217661

Sicard, A., Kappel, C., Josephs, E. B., Lee, Y. W., Marona, C., Stinchcombe, J. R., et al. (2015). Divergent sorting of a balanced ancestral polymorphism underlies the establishment of gene-flow barriers in Capsella. Nat. Commun. 6:7960. doi: $10.1038 /$ ncomms 8960

Singh, A., Taneja, J., Dasgupta, I., and Mukherjee, S. K. (2015). Development of plants resistant to tomato geminiviruses using artificial trans-acting small interfering RNA. Mol. Plant Pathol. 16, 724-734. doi: 10.1111/mpp.12229

Wulfert, S., and Krueger, S. (2018). Phosphoserine aminotransferasel is part of the phosphorylated pathways for serine biosynthesis and essential for light and sugar-dependent growth promotion. Front. Plant Sci. 9:1712. doi: 10.3389/fpls.2018.01712

Zhang, Z. J. (2014). Artificial trans-acting small interfering RNA: a tool for plant biology study and crop improvements. Planta 239, 1139-1146. doi: 10.1007/s00425-014-2054-x

Zhao, M., San León, D., Mesel, F., García, J. A., and Simón-Mateo, C. (2015). Assorted processing of synthetic trans-acting siRNAs and its activity in antiviral resistance. PLoS ONE 10:e0132281. doi: 10.1371/journal.pone.0132281

Zheng, X., Yang, L., Li, Q., Ji, L., Tang, A., Zang, L., et al. (2018). MIGS as a simple and efficient method for gene silencing in rice. Front. Plant Sci. 9:662. doi: $10.3389 /$ fpls.2018.00662

Conflict of Interest Statement: The author declares that the research was conducted in the absence of any commercial or financial relationships that could be construed as a potential conflict of interest.

Copyright (c) 2019 Carbonell. This is an open-access article distributed under the terms of the Creative Commons Attribution License (CC BY). The use, distribution or reproduction in other forums is permitted, provided the original author $(s)$ and the copyright owner(s) are credited and that the original publication in this journal is cited, in accordance with accepted academic practice. No use, distribution or reproduction is permitted which does not comply with these terms. 